\title{
NON-TUBERCULAR EMPYEMA THORACIS- A COMPARATIVE ANALYSIS OF GRAM POSITIVE AND GRAM NEGATIVE ORGANISM ASSOCIATED EMPYEMA
}

\author{
Subhasis Mukherjee ${ }^{1}$, Shabana Begum², Subhra Mitra3 ${ }^{3}$, Somenath Kundu ${ }^{4}$ \\ ${ }^{1}$ Assistant Professor, Department of Respiratory Medicine, College of Medicine and Sagar Dutta Hospital. \\ ${ }^{2}$ Demonstrator, Department of Anatomy, Medical College, Kolkata. \\ ${ }^{3}$ Professor, Department of Respiratory Medicine, Calcutta National Medical College. \\ ${ }^{4}$ Professor, Department of Respiratory Medicine, IPGMER and SSKM Hospital.
}

\begin{abstract}
BACKGROUND

Empyema thoracis is an entity resulting in significant morbidity and mortality. Bacterial empyema is the commonest cause of empyema globally. Clinical course and outcome of empyema varies in patients.

This study was carried out to evaluate clinical profile, diagnosis and outcome parameters of non-tubercular empyema cases with special interest to find out the differences between Gram positive and Gram negative organism associated empyemas in terms of clinical presentation and outcome issues.
\end{abstract}

\section{MATERIALS AND METHODS}

A prospective observational study of all cases of bacterial empyema thoracis admitted in the Department of Respiratory Medicine in a teaching institution over a period of 18 months was conducted. Out of the total no. of cases where pleural fluid culture was positive, a comparative analysis was done between cases of empyemas caused by Gram positive organisms and Gram negative organisms in terms of clinical characteristics, treatment and outcome issues.

\section{RESULTS}

A total no. of 95 empyema cases were encountered during the study period, of which fifty five were bacterial empyema. Pleural fluid culture was positive in $54.55 \%$ cases. Staphylococcus aureus $(15,27.3 \%)$ was the most frequent pathogen isolated. On comparative analysis of empyema cases caused by Gram positive and Gram negative organisms, it was found that Gram negative organism related empyemas was more frequent in older population and in people with co-morbidities. Clinical course of illness was more protracted and mean duration of intercostal tube drainage were longer (39.5 vs 17 days) in patients with Gram negative organism related empyemas. Presence of bronchopleural fistula was more in Gram negative empyemas and the need for surgical intervention was also higher in this group.

\section{CONCLUSION}

Bacterial empyema thoracis is the commonest cause of empyema. Gram negative organism related empyemas significantly differs from Gram positive organism related empyemas in terms of their clinical profile, increased occurrence of bronchopleural fistula and management issues.

\section{KEYWORDS}

Empyema Thoracis, Non-Tubercular Empyema, Gram Positive Organisms, Gram Negative Organisms.

HOW TO CITE THIS ARTICLE: Mukherjee S, Begum S, Mitra S, et al. Non-tubercular empyema thoracis- a comparative analysis of gram positive and gram negative organism associated empyema. J. Evolution Med. Dent. Sci. 2017;6(61):4445-4449, DOI: $10.14260 / \mathrm{Jemds} / 2017 / 961$

\section{BACKGROUND}

Bacterial empyema and tuberculosis account for the majority of empyema thoracis cases.[1.2] Empyemas caused by Gram negative organisms have been found to occur more in elderly people and people with diabetes mellitus.[3] In this background, this study was carried out to compare the clinical characteristics management and outcome issues between Gram positive and Gram negative organism related empyemas.

Financial or Other, Competing Interest: None.

Submission 11-07-2017, Peer Review 24-07-2017,

Acceptance 26-07-2017, Published 31-07-2017.

Corresponding Author:

Dr. Shabana Begum,

Mahendra Apartment,

181/2b, Roypur Road,

Kolkata-700047.

West Bengal.

E-mail:drs_begum@yahoo.com

DOI: $10.14260 /$ jemds $/ 2017 / 961$

(c) $(1)(-)$

\author{
MATERIALS AND METHODS \\ Study Design \\ Descriptive Study \\ A prospective, observational study of adult cases of non- \\ tubercular empyema thoracis admitted in the Department of \\ Respiratory Medicine of a teaching hospital in Eastern India \\ was carried out from January 2015 - June 2016.
}

\section{Patient Selection \\ Case Definition}

Empyema thoracis was defined as pleural effusion fulfilling one of the following criteria: - (1) Frank pus on pleural aspiration and/or (2) Growth of organism on pleural fluid culture and/or (3) Positive pleural fluid Gram stain.

\section{Exclusion Criteria}

(1) Tubercular empyema or probable tubercular empyema; (2) Age less than 15 years; (3) Empyema secondary to penetrating or blunt chest trauma; (4) Empyema secondary 
to any surgical procedure; (5) Patients not giving consent for the study.

Tubercular empyema was defined as the following for the purpose of exclusion from the study: (1) Pleural fluid Acid Fast Bacilli (AFB) smear positive or detection of Mycobacterium tuberculosis on Cartridge Based Nucleic Acid Amplification Test (CBNAAT) and/or (2) Sputum AFB smear positive or detection of Mycobacterium tuberculosis on CBNAAT and having radiological lesions consistent with active pulmonary tuberculosis on chest x-ray/CT scan of the thorax (consolidation with or without cavity, tree in bud appearance). Probable tubercular empyema was defined as empyema in patients who had only radiological evidence of active pulmonary tuberculosis on chest x-ray/CT scan of the thorax or were sputum AFB smear positive or CBNAAT positive, but pleural fluid AFB smear and CBNAAT were negative.

\section{Study Protocol}

All cases of non-tubercular empyema who fulfilled the inclusion criteria and had given consent for the study were subsequently analysed in respect to demographic parameters (age, sex, symptom duration, comorbidities). Chest x-rays were done in all patients on admission and also after Intercostal Tube Drain (ICTD) insertion, ICTD removal and at discharge. Ultrasound (USG) and contrast enhanced Computed Tomography (CT) of chest were done in selected cases. Pleural fluid was collected aseptically by thoracentesis and if macroscopically purulent was sent for Gram stain, culture (aerobic), smear for Acid Fast Bacilli (AFB) in RNTCP designated microscopy centre and CBNAAT for mycobacterium tuberculosis. Non-purulent fluid was also sent for cell type and cell count (total and differential), protein, sugar and LDH. Adenosine deaminase estimation was not done, as it can be elevated in empyema of any cause. Anaerobic culture was done in the clinical context of suspected anaerobic infections (foul smelling pus, h/o alcoholism, aspiration, poor orodental hygiene). Sputum was sent for AFB smear and CBNAAT in all cases to exclude tubercular empyema. Complete haemogram, renal and liver function tests, blood for HIV serology from integrated counselling and testing centre, blood sugar (fasting and postprandial) were obtained in all cases. A comparative analysis of Gram positive organism associated empyema and Gram negative organism associated empyema was carried out.

Bronchopleural Fistula (BPF) was suspected when chest $\mathrm{x}$-ray PA view before thoracentesis showed horizontal air fluid level and persistence of air bubbling through the intercostal tube drainage system beyond 24 hours after tube thoracostomy.

Intercostal Tube Drainage (ICTD) was carried out with a straight chest tube (Romson $28 \mathrm{~F}$ to $32 \mathrm{~F}$ ) without trocar with water-seal drainage system. In cases of encysted empyemas, where ICTD insertion was not feasible pigtail catheters (8.5Fr) was inserted under ultrasound guidance. In a few cases of multiloculated small empyemas, where neither ICTD nor pigtail drainage was feasible serial ultrasound-guided aspiration of pus was carried out. ICTD removal was considered when fluid was serous, daily collection became less than $50 \mathrm{~mL}$ without any air bubbling and CXR showed expansion of lung with acceptable pleural thickening. All patients of non-tubercular empyema were given antibiotics for four to six weeks- intravenous antibiotics for two weeks followed by oral antibiotics. Antibiotics were started empirically after sending the pleural fluid for culturePiperacillin-Tazobactam or a cephalosporin like cefoperazone-sulbactam/ cefepime-tazobactam and clindamycin or metronidazole were the initial antibiotics of choice for this study. Antibiotic regimen was modified subsequently based on the result of pleural fluid culture report. Aminoglycosides were avoided because of their poor concentration in pus and fluoroquinolones were also being avoided empirically because of high prevalence of tuberculosis. Intrapleural fibrinolytics was not used in the study as a result of negative result in MIST trial.[4] Inadequate drainage, persistence of BPF and/or non-expansion of the lung even after antibiotic therapy and intercostal drainage were referred to cardiothoracic surgeon and decortication and/or decortication with closure of bronchopleural fistula using intercostal muscle flap were performed in selected cases.

\section{Outcome}

All patients were followed up for three months. Outcome was defined as one of the following- Cure: complete lung expansion with minimum pleural thickening of $<2 \mathrm{~cm}$ in chest skiagram. Failure: Non-expansion or incomplete expansion of the lung and/or persistence of BPF after medical and surgical management. Death: Death within the study period due to the disease process.

\section{Statistical Analysis}

Statistical analyses were performed using SPSS version 20.0. Descriptive frequencies were expressed using mean. P value was calculated using Fisher's Exact test of significance for categorical variables and Independent Sample ' $t$ ' test for continuous variables and $\mathrm{P}$ value $<0.05$ was considered to be significant.

\section{RESULTS}

Ninety five patients of empyema thoracis were encountered during the study period, of which 40 (42.1\%) were of tubercular aetiology and were excluded from this study. Fifty five cases (57.9\%) of non-tubercular empyemas were considered for subsequent analysis.

\section{Bacteriologic Spectrum}

Out of these 55 cases of non-tubercular empyema, frank pus was seen on aspiration in 46 cases (83.64\%). Pleural fluid culture was positive in 30 cases (54.55\%), positive Gram stain but negative culture was seen in additional 9 cases $(16.36 \%)$ and pus or fluid was sterile in remaining 16 cases (29.09\%). Pleural fluid culture was positive in only 16 of the 36 patients $(44.44 \%)$ receiving antibiotics for more than 48 hours duration prior to admission, whereas culture positivity was found to be $73.68 \%$ in those receiving antibiotics for less than 48 hours duration before admission (Table 1). Staphylococcus aureus was found to be the commonest organism $(15,27.27 \%)$, closely followed by Gram negative bacilli (14, 25.45\%) (Table 2). Multidrug resistant Extended Spectrum $\beta$ Lactamase (ESBL) producing organisms were detected on culture sensitivity of pleural pus in three patients of Gram negative empyemas and methicillin-resistant Staphylococcus aureus (MRSA) was found in one case of Gram positive empyema. 
There was no significant difference in sex distribution with male predominance in both the groups $168.75 \%$ in Gram positive organism related empyema; $64.29 \%$ in Gram negative organism related empyema). Seventy five percent of Gram positive organism related empyemas were below 45 years of age in contrast to $64.29 \%$ of Gram negative organism associated empyemas in above 45 years age (Table 3). Mean age in the Gram positive organism associated empyema group was 32.7 years (range: 14 - 65) compared to mean age of 46.5 years (range: 13 - 72) Gram negative organism associated empyema group. No significant difference was noted between the two groups in relation to duration of illness, $75 \%$ of Gram positive organism associated empyemas and $70 \%$ of Gram negative organism associated empyemas were of less than one month duration ( $p-0.405)$. Five cases (35.71\%) of Gram negative organism associated empyemas had associated Bronchopleural Fistula (BPF) in contrast to no BPF in Gram positive organism associated empyemas ( $p$ 0.014 ). Diabetes mellitus was the commonest comorbidity in both the groups (25.45\%). It was seen in $43.75 \%$ and $50 \%$ cases of Gram positive and Gram negative organism associated empyema respectively. Gram negative empyema were seen in two patients of cancer receiving chemotherapy. Alcoholism, seizure disorder, Human Immunodeficiency Virus (HIV) infection and liver abscess were other comorbidities in this study.

\section{Radiology}

Large effusion ( $>50 \%$ of hemithoracic volume) was seen in 70.91\% cases (Figure 1), hydropneumothorax was seen in 9 cases (16.36\%) (Figure 2) and small encysted pleural effusion in $12.73 \%$ patients. Hydropneumothorax was found in $42.85 \%$ of the Gram negative empyema associated cases and $18.75 \%$ of Gram positive organism associated empyema cases.

\section{Treatment and Outcome}

Mean duration of intravenous antibiotic use was 20.5 days and mean duration of total (Intravenous + oral) antibiotic use was 36.2 days. In all cases, intravenous antibiotics were started after sending the pleural pus for culture. Antibiotics were then guided by the subsequent culture sensitivity report in $54.55 \%$ cases, empirical antibiotics were continued guided by clinical response in rest $45.45 \%$ cases. ICTD was inserted in 42 cases $(76.36 \%)$, pigtail catheter drainage was done in six cases $(10.91 \%)$ and serial USG guided aspiration of pus was done in seven cases (12.73\%), where ICTD or pigtailcatheter drainage were not possible. Mean duration of ICTD was significantly higher in Gram negative empyema group (39.5 days) compared to 17 days in Gram positive empyema group. Decortication with or without closure of BPF was needed in five patients of Gram negative empyemas, while none of the Gram positive empyemas required decortication (p- 0.0001) (Figure 3). There was good outcome in all five patients requiring decortication and no patient in either group died during the study period.

\begin{tabular}{|c|c|c|c|}
\hline $\begin{array}{c}\text { Duration of } \\
\text { Antibiotic use } \\
\text { prior to } \\
\text { Admission }\end{array}$ & $\begin{array}{c}\text { Pleural Fluid } \\
\text { Ordinary } \\
\text { Culture } \\
\text { Positive }\end{array}$ & $\begin{array}{c}\text { Pleural Fluid } \\
\text { Ordinary } \\
\text { Culture } \\
\text { Negative }\end{array}$ & P Value \\
\cline { 1 - 3 } $\begin{array}{c}<48 \text { hours } \\
(\mathrm{n}=17)\end{array}$ & 14 & 5 \\
$(73.68 \%)$ & $(26.32 \%)$ & \multirow{2}{*}{0.04} \\
\cline { 1 - 3 }$>48$ hours & 16 & 20 & \\
$(\mathrm{n}=38)$ & $(44.44 \%)$ & $(55.55 \%)$ & \\
\hline \multicolumn{3}{|c|}{ Table 1. Prior Antibiotic use and } \\
Pleural Fluid Culture Result \\
\hline
\end{tabular}

\begin{tabular}{|c|c|}
\hline Organism & Number (\%) \\
\hline Gram positive bacteria & $16(29.10 \%)$ \\
Staphylococcus aureus & $15(27.27 \%)$ \\
Streptococcus haemolyticus & 1 \\
\hline Gram negative bacteria & $14(25.45 \%)$ \\
Klebsiella pneumoniae & 5 \\
Pseudomonas aeruginosa & 3 \\
Proteus spp. & 2 \\
E. coli spp. & 1 \\
Enterobacter spp. & 1 \\
Citrobacter spp. & 1 \\
Acinetobacter spp. & 1 \\
\hline No growth on culture & $25(45.45 \%)$ \\
\hline Table 2. Bacterial Spectrum of \\
Non-Tubercular Empyema (n=55) \\
\hline
\end{tabular}

\begin{tabular}{|c|c|c|c|}
\hline $\begin{array}{c}\text { Age } \\
\text { (In Years) }\end{array}$ & $\begin{array}{c}\text { Gram Positive } \\
\text { Empyema } \\
\text { (n = 16) }\end{array}$ & $\begin{array}{c}\text { Gram Negative } \\
\text { Empyema } \\
\text { (n = 14) }\end{array}$ & P-Value \\
\hline $15-30$ & 8 & 2 & \multirow{2}{*}{0.063} \\
\hline $31-45$ & 4 & 2 & \\
\hline $46-60$ & 3 & 5 & 4 \\
\hline$>60$ & 1 & Table 3. Age Distribution \\
\hline \multicolumn{3}{|c|}{} \\
\hline
\end{tabular}

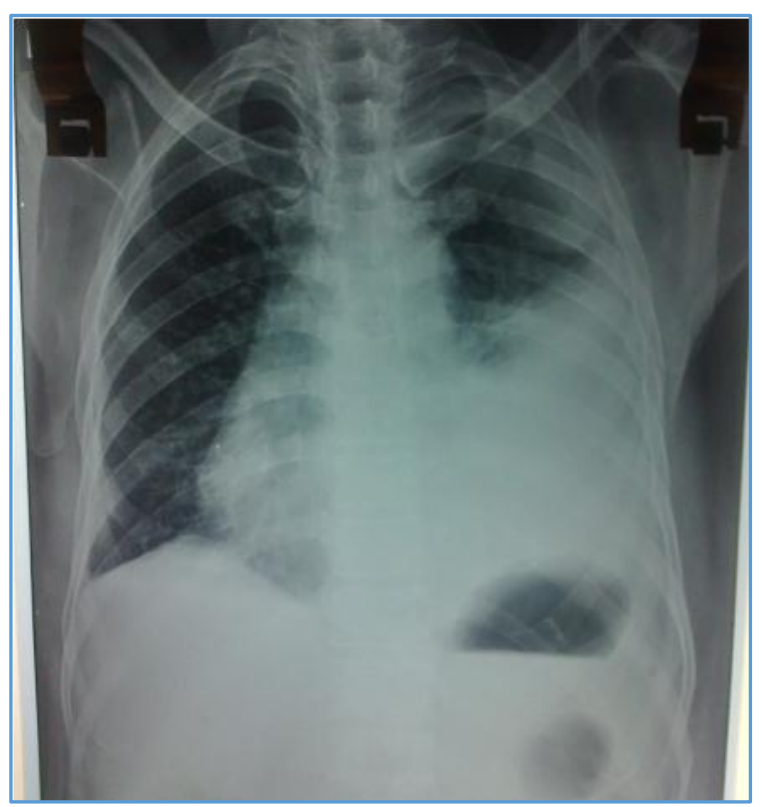

Figure 1. Chest X-Ray PA view showing Left-Sided Empyema 


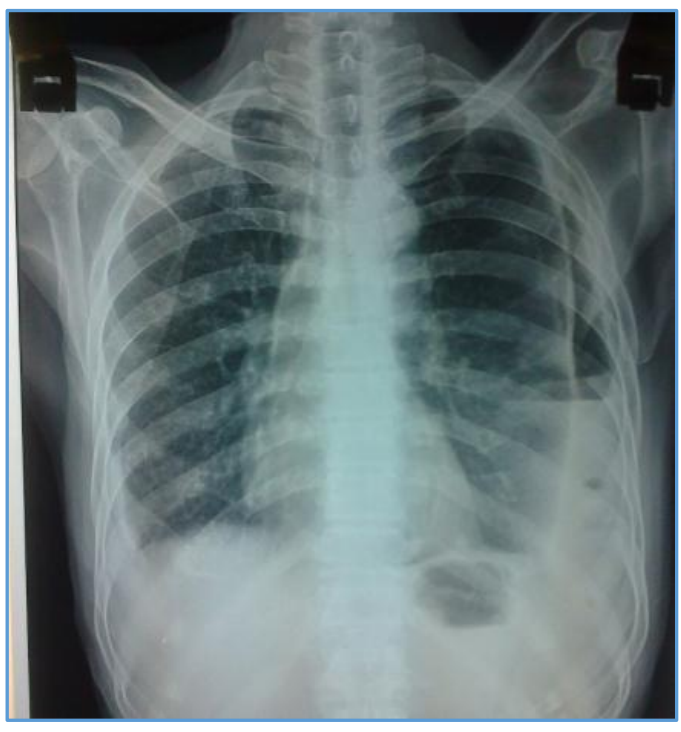

Figure 2. Chest X-Ray PA view showing Left-Sided Pyopneumothorax

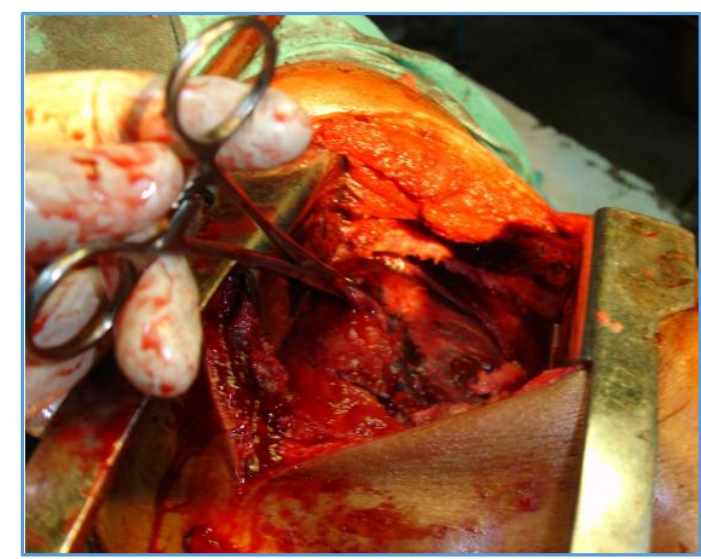

Figure 3. Per Operative Photograph during Decortication showing Thick Pleura adherent to underlying Lung Tissue

\section{DISCUSSION}

Bacterial infections are the commonest cause of thoracic empyema worldwide apart from post-surgical and posttraumatic causes,[1,2] although tubercular empyema is quite prevalent in developing countries.[2,5,6]

Microbiological diagnosis of empyema cases often become difficult as a result of frequent negative results of pleural fluid culture. In this study pleural fluid culture was positive in $54.55 \%$ cases and in about $29 \%$ patients both pleural fluid Gram stain and culture were negative. Kundu S et $\mathrm{al}^{[2]}$ Banga $\mathrm{A}$ et al[7] and Sethy $\mathrm{H}$ et $\mathrm{al}^{[8]}$ have reported pleural fluid culture positivity as $43.5 \%, 42 \%$ and $41 \%$ cases respectively in their studies. Prior antibiotic use has been shown to be an important cause for subsequent pleural fluid culture negativity.[2,8] Streptococcal pneumoniae associated parapneumonic effusions and empyemas are also frequently culture negative.[1] In this study, pleural fluid culture positivity varied significantly between patients receiving antibiotics for more than 48 hours before admission versus patients receiving antibiotics for less than 48 hours before admission ( $44.44 \%$ vs $73.68 \%$ ). This has been supported by previous study by Kundu S et al,[2] where yield of pleural fluid culture was $34.4 \%$ in patients receiving prolonged antibiotics and $64.2 \%$ in patients receiving antibiotics for less than 48 hours before admission.

Staphylococcal aureus was the commonest organism isolated in pleural fluid culture $(n=15,27.27 \%)$, closely followed by Gram negative bacilli ( $\mathrm{n}=14,25.45 \%$ ). Pseudomonas and Klebsiella were the two commonest Gram negative organisms isolated in this study. Other studies have also reported Staphylococcus aureus to be the commonest organism,[2,7] but increased occurrence of Gram negative organism associated empyemas has been reported in patients with comorbidities like diabetes mellitus by Begamy T et al..[3] In this study, diabetes mellitus was found to be the commonest comorbidity and was equally prevalent among both Gram positive organism associated (43.75\%) and Gram negative organism associated empyemas (50\%).

Gram positive organism associated empyemas usually affect younger age groups, whereas $64.29 \%$ of Gram negative organism associated empyemas were seen in above 45 years age group. In a previous study by Kundu S et al, $60 \%$ of Gram negative organism associated empyemas were reported in above 60 years of age.[2] Associated BPF was seen in 5 cases (28.57\%) of Gram negative organism associated empyemas. Similar results have been seen in other studies.[2,3] Initial chest x-ray showed hydropneumothorax in $45.85 \%$ cases of Gram negative organism associated empyemas.

Mean duration of ICTD was significantly higher in Gram negative organism associated empyemas compared to gram positive organism associated empyemas (39.5 days versus 17 days). Decortication with or without BPF closure was needed in five cases $(35.71 \%)$ of Gram negative empyemas in contrast to none in Gram positive organism associated empyemas. Begamy $\mathrm{T}$ et al have also reported about adverse prognosis in case of Gram negative organism associated empyemas. ${ }^{[3]}$ Delay in thoracic surgery like decortication has been shown to be adverse prognostic factor for empyema thoracis and it also increases chance of recurrent empyema thoracis. ${ }^{[9]}$ Decortication with closure of BPF using intercostal muscle flap and thoracoplasty are the standard thoracic surgical procedures usually used for thoracic empyema cases failing on medical management and intercostal drainage.[10,11] Video Assisted Thoracoscopy (VATS) guided decortication has also shown to be a promising option with the advantage of lesser morbidity and post-operative hospital stay compared to open thoracotomy, especially in elderly people and with Stage 1 and 2 empyema.[10,12]

Limitation of the study was culture negativity in about $45.45 \%$ cases, so actual sample size of Gram positive and Gram negative organism associated empyemas taken for comparison were small. However, a detailed and in-depth comparative analysis were tried, as there is paucity of published literature regarding difference in clinical behaviour of Gram negative and Gram positive organism associated empyemas. So, future studies comprising of larger sample size will be better for a more definitive and conclusive opinion.

\section{CONCLUSION}

In comparison of Gram positive organism associated empyemas, Gram negative organism associated empyemas are more commonly seen in elderly people with comorbidities, are significantly more associated with bronchopleural fistula, and frequently need prolonged 
intercostal chest tube drainage and complicated thoracic surgical procedures like decortication.

\section{REFERENCES}

[1] Light RW. Parapneumonic effusions and empyema. In: Rhyner S, Winter N, Koleth J, (eds). Pleural diseases. $5^{\text {th }}$ edn. Philadelphia: Lippincott Williams \& Wilkins 2007:179-210.

[2] Kundu S, Mitra S, Mukherjee S, et al. Adult thoracic empyema: a comparative analysis of tuberculous and nontuberculous etiology in 75 patients. Lung India 2010;27(4):196-201.

[3] Begamy T. Thoracic empyema. Is its microbiology changing? Pul Rev Com 2000;5:10.

[4] Maskell NA, Davies CW, Nunn AJ, et al. U.K. controlled trial of intrapleural streptokinase for pleural infection. N Eng J Med 2005;352(9):865-74.

[5] Malhotra P, Aggarwal AN, Agarwal R, et al. Clinical characteristics and outcome of empyema thoracis in 117 patients: a comparative analysis of tubercular vs. non tubercular aetiologies. Respir Med 2007;101(3): 423-30.

[6] Prakash B, Khare P, Bhatnagar AK. Tuberculous empyema thoracis: clinical, bacteriological features and its medical management. Int J Sci Stud 2015;3(6):120-5.
[7] Banga A, Khilnani GC, Sharma SK, et al. A study of empyema thoracis and role of intrapleural streptokinase in its management. BMC Inf Dis 2004;4:19.

[8] Sethy HK, Panda G, Trilochan BP, et al. Empyema thoracis: a current profile at a tertiary care center. J Evolution Med Dent Sci 2016;5(13):547-56.

[9] Tantraworasin A, Thepbunchonchai A, Siwachat S, et al. Factors associated with recurrent bacterial empyema thoracis. Asian Journal of Surgery 2017.

[10] Taylor MD, Kozower BD. Surgical spectrum in the management of empyemas. Thorac Surg Clin 2012;22(3):431-40.

[11] Nichkaode PB, Dasgupta S, Zamad R, et al. Open thoracotomy and decortication for chronic empyema (fibrothorax). Journal of Evolution of Medical and Dental Sciences 2013;2(17):2857-63.

[12] Tsai CH, Lai YC, Chang SC, et al. Video-assisted thoracoscopic surgical decortication in the elderly with thoracic empyema: five years' experience. J Chin Med Assoc 2016;79(1):25-8. 